Revue d'histoire de l'enfance « irrégulière »

Le Temps de l'histoire

1 | 1998

La protection de l'enfance : regards

\title{
Les centres d'accueil et de triage de l'Éducation surveillée : 1941-1950
}

Christian Sanchez

\section{(2) OpenEdition \\ 12 Journals}

Édition électronique

URL : http://journals.openedition.org/rhei/25

DOI : $10.4000 /$ rhei. 25

ISBN : 978-2-7535-1638-0

ISSN : $1777-540 \mathrm{X}$

Éditeur

Presses universitaires de Rennes

Édition imprimée

Date de publication : 15 novembre 1998

Pagination : 120-134

ISSN : 1287-2431

Référence électronique

Christian Sanchez, «Les centres d'accueil et de triage de l'Éducation surveillée : 1941-1950 », Revue d'histoire de l'enfance « irrégulière » [En ligne], 1 | 1998, mis en ligne le 30 avril 2007, consulté le 04 décembre 2020. URL : http://journals.openedition.org/rhei/25 ; DOI : https://doi.org/10.4000/rhei.25 
Centre d'accueil et de triage de Charenton

[1945-1949, archives du Centre d'observation

des mineurs de Charenton]

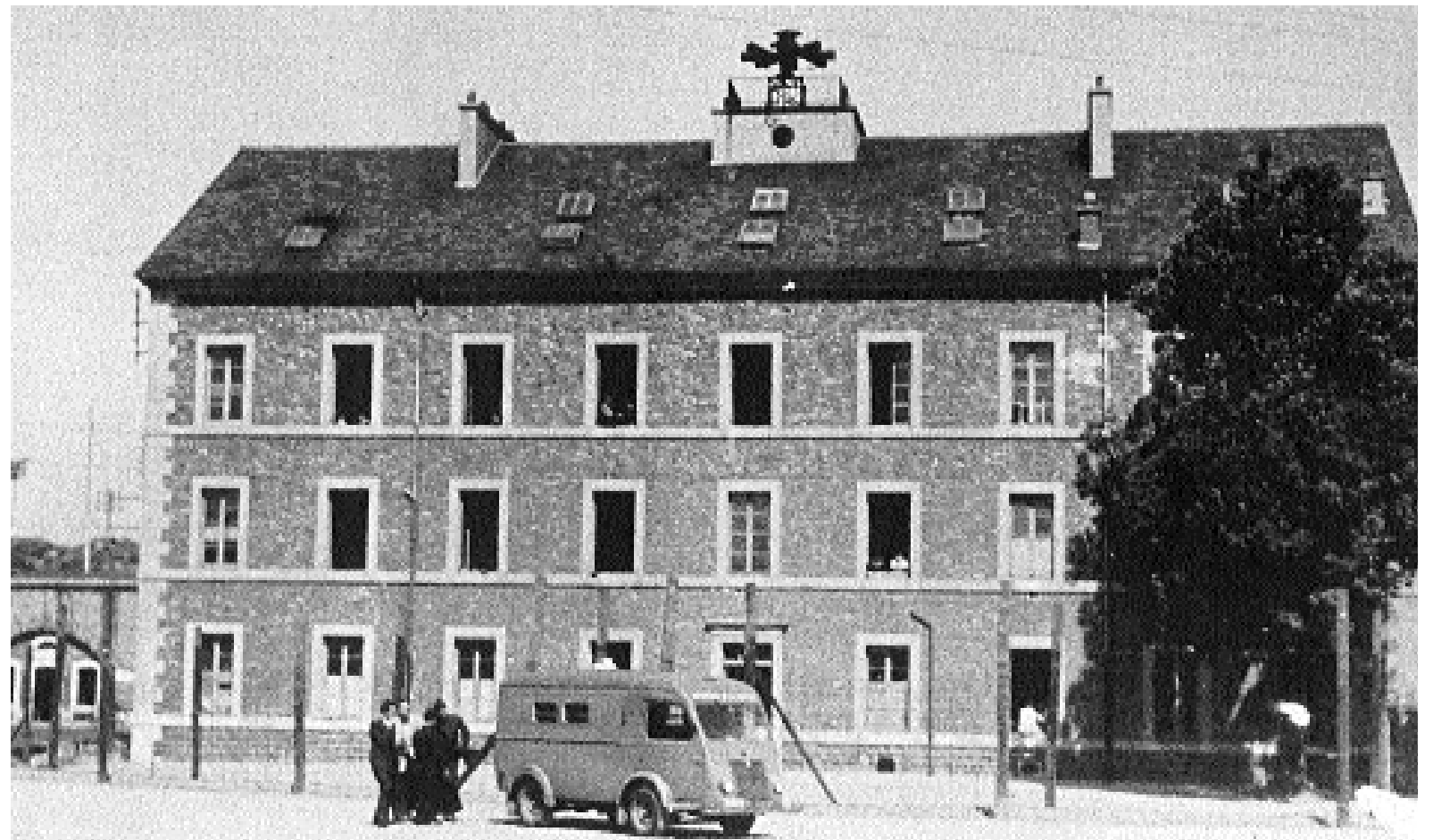

L'arrivée des pupilles en camion

cellulaire

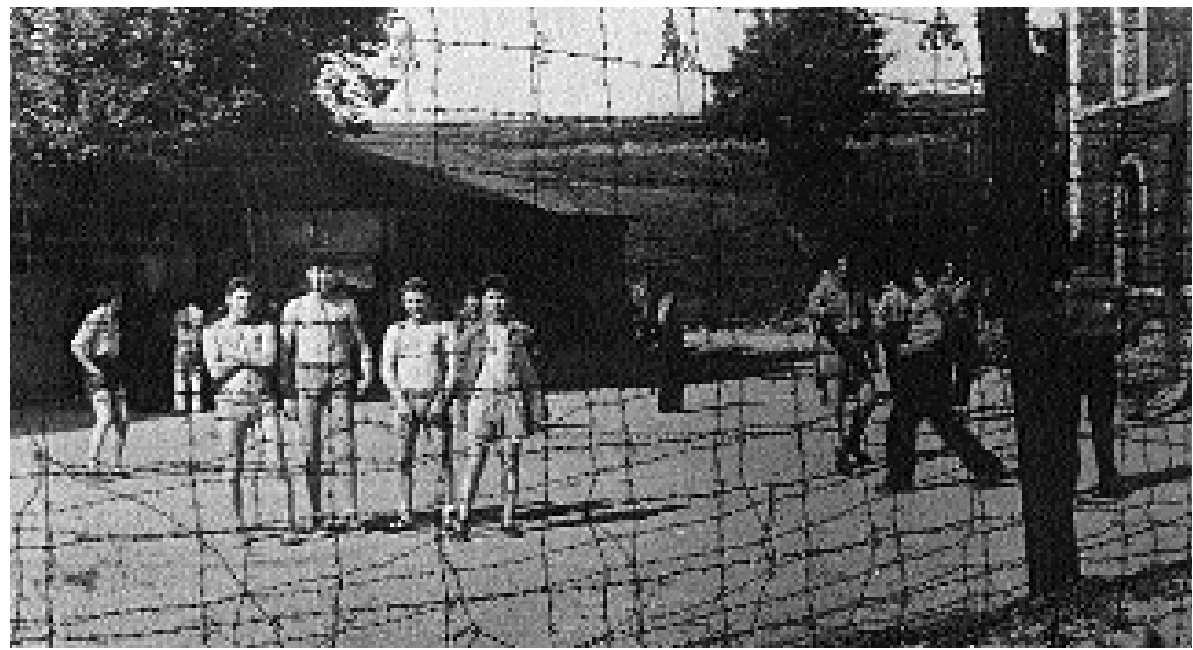

Mineurs

en observation 


\section{Les centres d'accueil et de triage de l'Éducation surveillée 1941-1950}

\section{Christian Sanchez ${ }^{(1)}$}

(1) Chef de service

éducatif à la Protection judiciaire de la jeunesse.
La loi du 27 juillet 1942 substitue à la notion de discernement celle d'éducabilité. Elle crée une nouvelle juridiction des mineurs dotée de magistrats spécialisés.

La nouvelle législation conjugue des impératifs à la fois économiques, sociologiques et idéologiques avec les attentes des professionnels de la "jeunesse irrégulière". Entre rupture et continuité cette loi s'inscrit dans un moment particulier de la France. Si elle permet de s'orienter, semblet-il, définitivement sur la route de la primauté de la mesure éducative, il n'en demeure pas moins qu'elle comporte les moyens d'un retour au pénitentiaire, notamment à l'aide des colonies correctives qu'elle prévoit pour les mineurs les plus difficiles. Cependant, elle instaure en France ce qui se pratique depuis plusieurs années en Europe, l'individualisation de l'action éducative reposant sur l'observation du mineur.

En 1942, le patrimoine de l'Éducation surveillée, simple service de l'administration pénitentiaire, est des plus archaïques en matière d'établissements. Il n'existe pas d'institution répondant aux exigences de la loi. On fera appel à l'initiative privée et au secrétariat à la jeunesse. Le service public organisera son infrastructure autour d'un pivot institutionnel, le centre d'observation. Ce dernier ne verra toutefois le jour qu'en 1945. Entre temps, quelques centres d'accueil et de triage seront créés. La hâte, l'hésitation idéologique, méthodologique semble caractériser ces établissements.

Les centres d'accueil et de triage n'ont-ils été qu'un épisode dans une chronologie de la rééducation triomphante? Une parenthèse créatrice? Un polissoir de logiques et de techniques visant à créer les conditions 
(2) Il ne s'agira ici que des réalisations de la région parisienne.

(3) Michel Foucault, Surveiller et punir, p. 254.

(4) Le discernement : "L'intelligence légale qu'un individu est censé avoir de la criminalité de l'action qu’il a commise ». Définition donnée par le rapport de l'Administration pénitentiaire de France au congrès international de Bruxelles (1900). C'est l'article 66 du code criminel (1791), repris dans le code pénal (1810), qui stipule :

"Lorsqu'un accusé, déclaré coupable par le jury, aura commis le crime pour lequel il est poursuivi avant l'âge de 16 ans accomplis, les jurés décideront, dans les formes ordinaires de leur délibération, la question suivante : le coupable a-t-il commis le crime avec ou sans discernement?"

(5) L'article V du code criminel de 1791 précise que « toute maison de correction sera une maison de de réussite d'une politique hardie en matière de délinquance juvénile? Sans prétendre répondre à ces vastes questions, il s'agit ici de situer les centres d'accueil dans l'histoire de la rééducation, d'appréhender les réalisations ${ }^{(2)}$ et de tirer quelques pistes de réflexion.

\section{QUELQUES ÉLÉMENTS D'HISTOIRE}

À la fin du XVIIIème siècle, l'enfant est moins considéré comme un adulte en réduction. Il devient peu à peu la personne autour de laquelle va se construire la famille. La Révolution française entraîne des transformations socio-économiques. Le travail devient l'une des valeurs fondatrices de la nouvelle société. Tous les bras seront nécessaires; il ne faudra écarter aucun individu; ceux qui auront commis des délits devront être non seulement punis mais également rééduqués et réinsérés. C’est à cette époque que va naître le concept de délinquance. Michel Foucault précise que "le délinquant se distingue de l'infracteur par le fait que c'est moins son acte que sa vie qui est pertinente pour le caractériser $\gg{ }^{(3)}$ C'est donc par l'enquête biographique que l'on va pouvoir déterminer la personnalité du délinquant, le rendre visible et prévisible.

Dans la première république, qui se voulait éducatrice de la jeunesse, la justice pour mineurs reposera sur la notion de discernement. ${ }^{(4)}$ Le mineur reconnu discernant sera écarté de la voie "éducative" et condamné. Non discernant, il sera conduit dans une "maison de correction". ${ }^{(5)}$ Dès l'entrée du colon dans l'institution, l'enquête biographique commence; une notice est établie à la demande du ministère de l'Intérieur. On ne peut pas parler d'observation. Mais curieusement les rapports d'observation rappelleront, par bien des points, les notices sur lesquelles étaient transcrites des informations sur les antécédents familiaux, sur le mineur, sur sa scolarité, etc... Le regard était essentiellement un regard disciplinaire. Il avait pour objectif de maintenir l'ordre par un "dressage qui s'accompagne d'une observation permanente sur la conduite quotidienne $d u$ colon. Un savoir est sans cesse prélevé ». ${ }^{(6)}$ Michel Foucault nous fait remarquer que, pendant les journées révolutionnaires de 1848, aucun trouble n'avait été signalé à la colonie agricole de Mettray. ${ }^{(7)}$ La loi du 5 août 
1850 relative à l'éducation et au patronage des jeunes détenus n'apporte guère d'éléments en matière d'observation.

La loi du 24 juillet 1889 concernant l'enfance maltraitée ou moralement abandonnée va permettre de confier les mineurs pendant le temps de l'instruction à une personne digne de confiance ou à une institution charitable. Plus tard, une subtile modification rédactionnelle ${ }^{(8)}$ de la loi de 1898 "sur la répression des violences voies de fait, actes de cruauté et attentats commis envers les enfants " permit d'assimiler les enfants auteurs de délits aux enfants victimes et, par là, de bénéficier de l'article permettant de les confier à un parent, une personne digne de confiance, une institution charitable. Ces deux lois vont permettre l'éclosion d'un nouveau type de patronage privé que l'on qualifiera d'“ouvert" par rapport à ceux de la pénitentiaire. Ces sociétés de patronage se livrèrent, sur les classes pauvres, à un véritable travail ethnographique. Elles se voulaient les paires de lunettes et les yeux de l'administration. ${ }^{(9)}$ La pratique de ces sociétés, qui allaient préfigurer le milieu ouvert, annonçait un nouveau métier, "l'assistante sociale", dont l'enquête du même nom sera une technique fondatrice de l'observation des mineurs. Les textes de 1889 et de 1898 ne prévoient cependant pas de profession spécifique et «se contentent d'enquêtes sommaires faites par les auxiliaires habituels du parquet, c'est-à-dire les services de police avec parfois l'intervention du juge de paix $»{ }^{(10)}$

Par ailleurs, la criminologie se développe en Europe avec l'école italienne (Lombroso). L'école française de criminologie, avec Lacassagne, en désaccord avec les théories de Lombroso, préconise la prévention. Elle souligne les effets bénéfiques de l'éducation sur l'enfant "vicieux". L'évolution des sociétés de patronage répondra alors aux attentes de la jeune science criminologique, qui n'a de cesse de demander dans ses congrès l'étude préalable de la personnalité du mineur, notamment à l'aide de l'examen médico-psychologique.

La loi du 22 juillet 1912 relative aux tribunaux pour enfants et adolescents et à la liberté surveillée n'aura pas de véritable effet sur les méthodes et le traitement des jeunes délinquants. Appliquée très partiellement, la loi n'offrira pas les moyens d'une nécessaire réforme des institutions existantes. Toutefois, on peut souligner son apport en matière de travail».

(6) cit., p 301.

(7) Mettray est la colonie agricole emblématique du XIXème. Sa célébrité égale sa longévité : 1839-1939.

(8) C'est le sénateur Bérenger qui fit modifier la rédaction, «en visant les crimes commis par des enfants » ou «sur des enfants». H. Gaillac, p. 244.

(9) Voir à ce sujet les travaux de Dessertine D. et Maradan B., en particulier « Pratiques judiciaires de l'assistance éducative (1889-1941)", Rapport intermédiaire de recherche au CNFEPJJ, Vaucresson, 1997.

(10) Henri Gaillac, Les maisons de correction, p. 329. 
(11) Ce service prendra le nom d'association Olga Spitzer.

(12) Un instituteur de Fresnes, Meurillon, relate de manière très précise ces activités d'observation dans une monographie. Arch. CNFE, Vaucresson. dépistage et d'orientation. En instituant l'enquête sociale et en préconisant l'examen médico-psychologique, elle instaure une nouvelle dynamique. Le tribunal pour enfants et adolescents de la Seine va s'attacher les services de médecins s'inscrivant dans le jeune courant de neuropsychiatrie infantile et du service social à l'enfance. Cette collaboration aura pour objectif la recherche des causes de la délinquance juvénile.

À la même époque dans un pays voisin (Belgique), une législation permet la création d'un centre d'observation (Moll). L'observation repose sur l'enquête sociale, l'examen médico-psychologique et l'observation du comportement. Ce centre et sa méthode allaient devenir la référence européenne en matière d'observation des mineurs. Avant la Belgique, aux États Unis, on peut noter la création, en 1899, des "Child Guidance Clinics" et, en 1909, de l'institut psychologique du tribunal de Chicago. À Boston, c'est la célèbre consultation du Docteur Healy qui s'organise.

Les premières expériences françaises se mettent en place à partir du tribunal pour enfants et adolescents. Ce sera, en 1927, l'œuvre de "La titulaire", à Issy-les-Moulineaux, recevant les jeunes filles de 14 à 18 ans. Un an plus tard, le service social à l'enfance en danger ${ }^{(11)}$ ouvrira à Brunoy, en Seine-et-Oise (actuellement Essonne), le centre de Soulins pour jeunes enfants (garçons et filles). Enfin, le 19 janvier 1929, un décret va permettre non pas l'observation mais le triage des mineurs. En 1930, c'est sous une forme déjà très élaborée qu'une expérience d'observation verra le jour à la prison de Fresnes dans les locaux de la maison d'éducation surveillée. ${ }^{(12)}$

En 1935, la loi du 30 octobre dépénalise le vagabondage et exige que soit effectuée une enquête sociale et un examen médical avant la comparution devant chambre du conseil. Cette même loi va prévoir le placement des mineurs en attente de l'audience, dans un dépôt spécial. C'est à cette époque qu'apparaît le vocable de centre d'accueil, qui viendra compléter celui de centre de triage. Si une circulaire du garde des Sceaux de 1936 parle d'institution d'accueil d'observation et de triage, rien n'est cependant prévu pour leur fonctionnement.

La période de la seconde guerre mondiale sera propice aux expériences éducatives dans le domaine de l'enfance délinquante. La jeunes- 
se est au cœur des préoccupations du gouvernement de Vichy, dans un souci qui évolue entre endoctrinement et encadrement. La jeunesse délinquante n'y échappera pas.

Le 21 mars 1942, le Garde des sceaux promulgue une circulaire capitale relative à la détention préventive des mineurs. Elle souligne la volonté politique de s'orienter vers des accueils en milieu éducatif durant l'instruction. Cette circulaire, dont on attribue la rédaction à $P$. Ceccaldi, ${ }^{(13)}$ préconise aux procureurs généraux d'inviter les : « substituts à rechercher, au siège du tribunal ou dans les environs immédiats, un établissement public ou privé, ou encore une personne charitable, susceptible de prendre en charge l'enfant en attendant que l'autorité judiciaire statue sur son cas ». C'est ainsi que l'on pourra faire "appel sans qu'aucune habilitation spéciale à cet effet soit nécessaire, aux centres provisoires d'accueil, aux oeuvres privées de patronage, aux centres urbains et ruraux de jeunesse, aux organismes scolaires, ainsi qu'au zèle des personnes charitables et des femmes qui, en tant que membres d'assemblées municipales ou départementales, sont plus particulièrement chargées des questions d'assistance et d'enfance ".

Cette circulaire traduit la grande nécessité de places d'accueil pour mineurs. Bien sûr, le nombre de la délinquance augmente : de 1936 à 1942, il passe de 10879 à 34811, encore qu'une étude sur la nature des délits ${ }^{(14)}$ pourrait mieux éclairer la situation. Le problème de la jeunesse moralement abandonnée est préoccupant. La circulaire permettra une meilleure application de la loi du 27 juillet 1942 relative aux tribunaux pour enfants et adolescents et aux centres d'observation. Cette loi se veut être une rupture. Elle réforme la législation pénale, la procédure et abandonne la notion de discernement. Elle institue les tribunaux pour enfants et les centres d'observation.

La singularité de cette législation réside dans le fait qu'elle instaure des compétences juridictionnelles différenciées pour les mineurs de dixhuit ans. La chambre du conseil du tribunal civil avait une compétence d'arrondissement. Cette juridiction pouvait décider soit la remise de l'enfant à sa famille ou gardien, soit le renvoi de l'affaire devant le tribunal pour enfants. Ainsi, cette première approche permettait la sélection des affaires et le triage des mineurs. Ce triage était effectué dans les

(13) P. Ceccaldi est à l'époque chef de service de l'Éducation surveillée à l'Administration pénitentiaire. Il deviendra plus tard sous-directeur, puis directeur de l'Éducation surveillée de 1956 à 1964.

(14) La qualification des délits est parfois changée, notamment pour les mineurs résistants : un sabotage ferroviaire est qualifié de chapardage de charbon, dira un éducateur du centre de Villejuif. 
Centre d'accueil et de triage de Charenton

[1945-1949, archives du Centre d'observation des mineurs de Charenton]

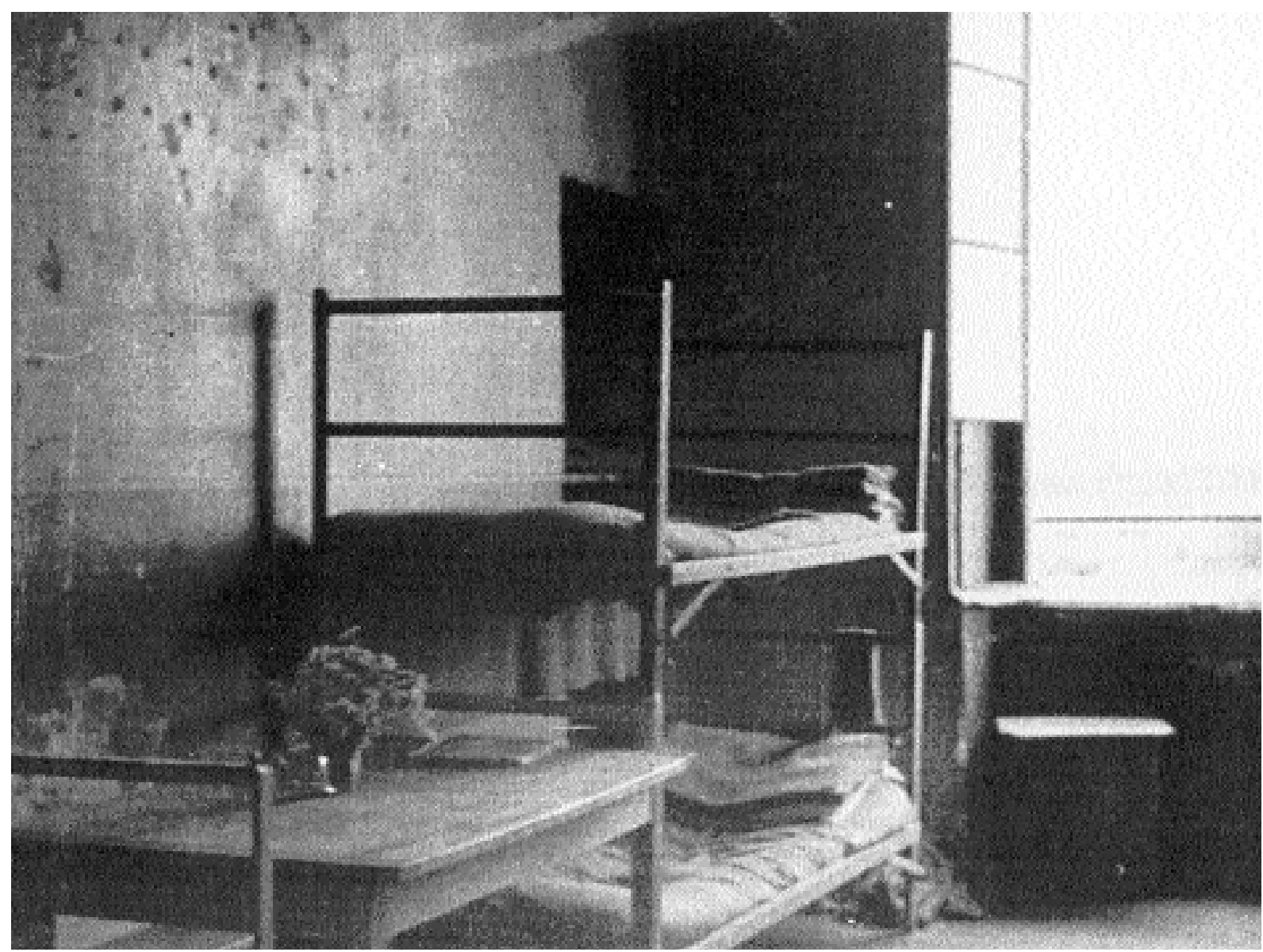

Une chambre

du centre de triage 


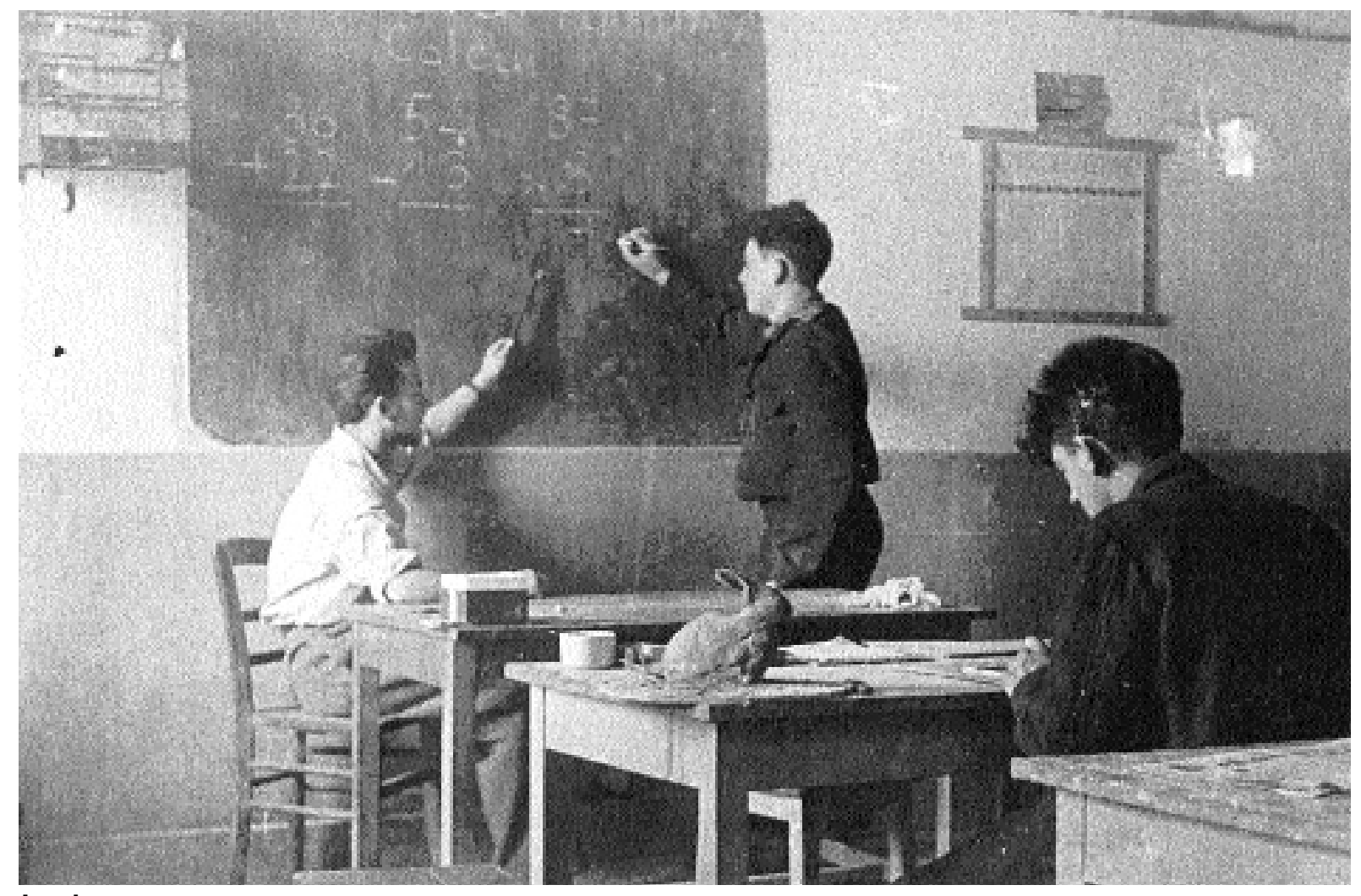

\section{La classe}

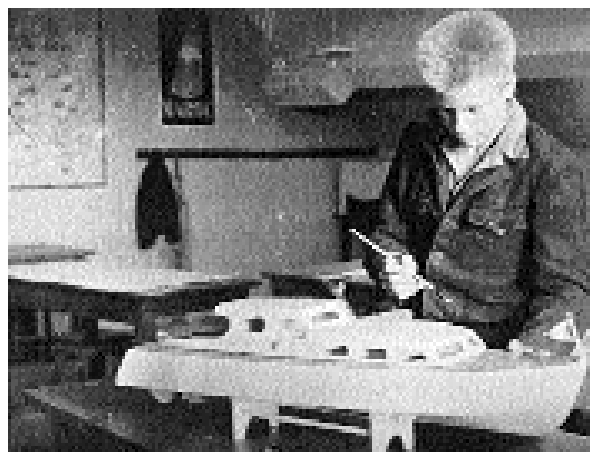

Atelier maquette 
(15) J. Pinatel, Précis de science pénitentiaire, p. 287.

(16) F. Dhallenne, Rapport à M. l'Inspecteur général Pinatel, p. 2, Arch. CNFE-PJJ, Vaucresson. centres d'accueil et de triage. Le tribunal pour enfants, quant à lui, avait une vocation régionale pour des affaires nécessitant une meilleure approche de la personnalité du mineur. Cette étude s'effectuait dans le centre d'observation, dans lequel il était prévu que siège le tribunal pour enfants. Une lacune est relevée par Jean Pinatel : "La chambre du conseil du tribunal civil, organe de solution et de triage, n'est pas composée de magistrats spécialisés. Par contre, le tribunal régional pour enfants et adolescents comprend des magistrats spécialisés. Il est présidé par un magistrat de cour d'appel ». ${ }^{(15)}$

Cette loi complexe ne sera pas ou sera peu appliquée mais, selon ses dispositions, des institutions verront le jour : les centres d'accueil et de triage. Ces institutions seront parfois jugées très sévèrement par les pionniers de l'Éducation surveillée : "Il n'est pas exagéré de prétendre que la création de ces derniers établissements n'a été qu'une suite d'improvisations bâtives dictées par les circonstances du moment et probablement sous la pression de certains personnages de Vichy qui ignorent totalement ou, voulant ignorer les réformes en cours depuis l'avant-guerre, reprenaient à zéro tout le problème de l'enfance délinquante ". ${ }^{(16)}$

Ce bref historique permet d'appréhender la dynamique dans laquelle se situaient les centres d'accueil au moment de leur création

À partir de 1942, l'organisation de la détention provisoire des mineurs durant l'instruction va s'effectuer en deux temps : le triage et l'observation.

\section{LES ÉTABLISSEMENTS DE TRIAGE}

Lorsque le mineur est interpellé, il est emmené au commissariat d'arrondissement. Il y passe un ou deux jours, mêlé aux adultes hommes et femmes. Il sera ensuite transféré au “dépôt” dans l'attente (quarante-huit heures) de sa comparution devant le magistrat instructeur. Selon la décision il sera confié, via la prison de la Santé, à un centre de triage. Dès leur arrivée, les mineurs sont dirigés vers le greffe, où ils sont immatriculés (registre d'écrou). On établit une fiche anthropométrique, puis ils vont "aux douches". On leur remet une serviette et un demi morceau de savon. Les douches sont froides. "La toilette est complétée par une mesure de 
désinfection corporelle. Un agent enduit chaque mineur d'un produit désinfec(17) À l'acide de bentant, particulièrement la tête ". ${ }^{(17)} \mathrm{Au}$ sortir de la douche, les mineurs trouveront l'uniforme du centre à la place de leurs vêtements, une tenue bleu, façon aviateur, qui était celle des chantiers de jeunesse. Ils seront alors examinés par le médecin qui établira leur carnet médical. Puis ils seront répartis dans les groupes selon les places disponibles. Le mineur partagera son temps, dans le meilleur des cas, entre la classe et l'éducation physique, en attendant son affectation dans l'un des centres d'accueil ou son jugement.

\section{La Maison d'éducation surveillée de Fresnes}

Le triage des mineurs s'effectuait préalablement à la MES de Fresnes. En 1940, les Allemands réquisitionnent les 240 cellules qui leur étaient réservées. Les mineurs sont entassés à trois ou quatre dans les cent cellules d'un quartier pour majeurs. Cette situation présidera à la création d'un service pour désengorger la MES de Fresnes (le centre de Crimée). ${ }^{(18)}$ Mais, très vite, la capacité d'accueil de ce nouveau centre ne sera pas suffisante.

\section{Le centre des Tourelles (1942-1945)}

À partir du 9 octobre 1942, on enverra les mineurs prévenus relevant de la juridiction de la Seine dans une caserne : une véritable prison. C'est dans des locaux de la caserne des Tourelles, peu adaptés, baptisés Maison d'éducation surveillée, que vivront dans l'oisiveté ${ }^{(19)}$ des centaines de mineurs envoyés là pour un premier triage, mais aussi pour une illusoire observation.

\section{Le centre de Charenton (1945-1949)}

Le 15 octobre 1945, dans un bâtiment du fort de Charenton gardé jour et nuit par des élèves-gendarmes, s'installe le nouveau centre qui présidera au triage des mineurs jusqu'au 1 juillet $1949 .{ }^{(20)}$

\section{Le centre de Madrid (1945-1948)}

Le 1er mars 1945, l'administration va implanter un groupe de triage au quatrième étage du centre d'accueil de la rue de Madrid. 
Ainsi, les premiers contacts du mineur avec l'éducation surveillée d'avant 1945 s'effectueront sous le signe de la répression dans les sinistres locaux d'une prison, d'une caserne, d'une école de gendarmes (entourée barbelés et surmontée de miradors). Avec l'ordonnance du 2 février1945, la pratique du triage cessera. Le placement en centre d'observation deviendra une des mesures éducatives que pourra prononcer le juge des enfants.

\section{LES ÉTABLISSEMENTS D’ACCUEIL ET D’OBSERVATION}

L'observation des mineurs débute dans un groupe dit d'accueil. Dès son arrivée, on demande au mineur d'exécuter un dessin libre et une rédaction dont le sujet est toujours à caractère autobiographique (la plus grande joie, la plus grande peine, etc.). Les travaux sont conservés dans "le cahier". Le chef de la section d'accueil lui fait passer un test permettant de mesurer son niveau mental, qui servira à l'affecter au bout de huit jours dans un groupe d'observation approprié. Le temps de l'accueil sera celui de l'investigation (sociale, somatique, psychiatrique).

Une fois arrivé au groupe d'observation, le mineur partage son temps entre la classe, l'atelier, la gymnastique et les veillées; tous ces moments deviendront les temps forts de l'observation directe du mineur.

Ce travail fournira, venu le temps de la comparution devant la juridiction, une fiche de renseignements succincte mais complète, portant sur :

$1^{\circ}$ les observations faites par le personnel d'éducation sur la conduite et le comportement du mineur au centre;

$2^{\circ}$ les renseignements d'ordre médico-psychologique;

$3^{\circ}$ les renseignements sociaux;

$4^{\circ}$ les relations de l'enfant avec sa famille;

$5^{\circ}$ les désirs de l'enfant;

$6^{\circ}$ une proposition de placement.

\section{Le centre d'accueil de la rue de Crimée (1941-1947)}

Créé le 1er avril 1941 pour "désengorger" la MES de Fresnes, l'établissement est un ancien orphelinat maçonnique réquisitionné par la préfecture de Paris et cédé à l’Administration pénitentiaire. Il va présider à 
l'observation des mineurs. Sa capacité est de 110 places. Il est bien adapté à sa nouvelle destination. Il fermera ses portes en mai 1947 et fusionnera avec le Centre d'observation de Savigny-sur-Orge.

\section{Le centre d'accueil de Villejuif (1943-1950)}

Ce centre, qui occupe deux quartiers (les 5 et 6) de l'hôpital psychiatrique de Villejuif, a été ouvert pour "soulager" la prison des Tourelles. Sa capacité d'accueil est de 80 garçons. Ce centre est considéré, malgré son implantation et la grande pénurie de ses moyens, comme le meilleur établissement d'observation de l'époque.

\section{Le centre de la rue de Madrid (1943-1948)}

Le centre s'installe dans les locaux d'un ancien collège tenu par les pères Jésuites. Il en possède tous les défauts, de longs couloirs tristes et une cour encaissée entre de hauts immeubles. Au printemps 1943, le ministère de la Justice confie au secrétariat général de la jeunesse l'organisation de ce centre (selon l'article 27 de la loi de 1942). Les frais d'entretien des prévenus sont en partie couverts par le ministère de l'Intérieur (qui succède au ministère de la Justice). Le personnel et le centre sont entièrement gérés par le secrétariat général à la jeunesse. En 1945, l'Éducation surveillée "récupérera les locaux" et poursuivra l'observation des mineurs. Sa capacité d'accueil est alors de 80 mineurs. En mars 1945, l'administration y logera un centre de triage d'une capacité d'accueil de 25 mineurs.

\section{LE POIDS DES LOIS, LE CHOC DE LA RÉALITÉ}

La loi est promulguée, la volonté politique exprimée, mais les moyens ne suivent pas. Comme dans les années trente, les budgets ne sont pas à la hauteur des objectifs poursuivis. Les moniteurs (surveillants) semblent encore veiller à la surveillance des enfants et à celle des moniteurséducateurs, perpétuant le syndrome de la réforme échouée de SaintMaurice, accentué par le décret du 31 août 1940 rétablissant l'interpénétration entre personnel de la pénitentiaire et de l'Éducation surveillée (au centre des Tourelles, par exemple, on compte 11 surveillants pour 6 
CNFE, Vaucresson, p. 4).

(21) Ce sera le statut des personnels de l'Éducation surveillée du 10 avril 1945. Voir J.

Bourquin, « Un statut qui précède le métier... ", dans ce numéro.

(22) M. Buzenac, Rapport sur le fonctionnement des groupes au centre de Madrid, 17 février 1945, Arch. CNFE, Vaucresson. moniteurs-éducateurs). À la Libération, la majorité des moniteurs-éducateurs, recrutés pendant l'occupation et dont l'une des motivations était d'échapper au STO, quittent l'Éducation surveillée pour rejoindre leurs activités antérieures.

La situation est critique. Fallait-il garder le personnel de la pénitentiaire? Mais que faire alors des grandes espérances pour l'observation? Le centre d'observation de l'Éducation surveillée allait s'ouvrir avec quel personnel ? L'administration tranchera en créant un corps d'éducateur de l'Éducation surveillée. ${ }^{(21)} 398$ personnels relevant de l'Administration pénitentiaire seront retenus, dont 160 le seront provisoirement. Le recrutement s'effectuera localement jusqu'en 1950, date à laquelle le premier concours prévu statutairement aura lieu.

Alors les centres d'accueil et de triage allaient connaître bien des avatars. Les espoirs d'œuvrer à un renouveau, le volontarisme scout, la mystique des chefs sont mis à rude épreuve par cette précarité dont on ne voit pas bien comment on en sortira. À cette pénurie s'ajoutent les difficultés inhérentes aux réquisitions successives des armées, à la fermeture de centres ou à des ouvertures improvisées, comme celle du centre de triage de la rue de Madrid, qui laissent bien amers les acteurs : "La première impression causée tant sur le personnel que sur les pupilles par le nouveau centre a été déplorable. Chacun s'est démoralisé par l'aspect rébarbatif de la nouvelle maison et l'état véritablement sordide dans lequel nous avons trouvé les lieux qui allaient devenir notre demeure ". ${ }^{(22)}$

À cette situation de nomadisme institutionnel, s'ajoute une situation alarmante. Les arrivants affluent; on compte 27 arrivants en une seule semaine au centre de Madrid. Cet établissement, qui ne devait recevoir que 90 mineurs, accueille 180 mineurs en moyenne. Au mois de septembre 1945, le doute s'installe. On sait les centres de Crimée et des Tourelles complets et on constate avec dépit que "Savigny, limité jusqu’à présent à deux pavillons, ne nous soulage guère davantage, et n'accepte d'ailleurs de recevoir que des catégories très déterminées de délinquants". Alors on peut comprendre certains privilèges, mais "nous ne pouvons continuer à accepter sans limitation, au prix d'un écroulement de la maison dont nous avons la charge, de recevoir le tout venant des jeunes prévenus de Paris et de sa banlieue ". 
Nous avons évoqué ici le centre de Madrid, mais tous les centres d'accueil et de triage étaient logés à la même enseigne. Ainsi, au centre de Charenton, on se réjouit que les douches fonctionnent (à l'eau froide), que la plupart des vitres aient été posées (au mois de janvier) et que l'on ait reçu des poêles à charbon mais que, si on a bien pu récupérer des tuyaux dans les casemates des alentours, il manque des coudes... Au mois de décembre 1950, après le centre de Crimée (1947), de Madrid (1948), de Charenton (1949), le centre de Villejuif ferme à son tour, tournant la page d'une histoire singulière, celle des centres de d'accueil et de triage de la région parisienne.

La chronologie de fermeture avait-elle été préparée? Nous ne pouvons l'affirmer. Il n'en demeure pas moins que "la politique de nondécision" qui apparaît de l'extérieur semble conduire les centres dans une sorte d'impasse, un bassin de décantation des techniques et des difficultés. Les cinq ans qu'il aura fallu, depuis la promulgation de l'ordonnance de 1945, pour aboutir à la fermeture des centres d'accueil auront été le temps de la mise à l'épreuve de la pluridisciplinarité, de la résorption du personnel de la pénitentiaire et de la création d'un corps d'éducateur. Bouc-émissaire chargé de toutes les erreurs précédentes, les centres d'accueil ont sans doute permis, par leur mort annoncée, d'imaginer une nouvelle virginité au centre d'observation de l'Éducation surveillée de Savigny-sur-Orge, modèle français de l'observation scientifique. Il pouvait alors prospérer et imposer sa méthode et son nouveau règlement à l'ensemble de l'enfance inadaptée.

\section{Éléments de bibliographie}

BOURQUIN (J.) \& KEPPEL (B.), "Deux contributions à la connaissance des origines de l'Éducation Surveillée”, Cabiers $d u C R I V, \mathrm{n}^{\circ} 2$, Vaucresson, 1986, 93 p.

BRUNEL (J.), Souvenir d'un éducateur, Paris, Le pied à l'étrier, 1967, 93 p. CHAUVIËRE (M.), Enfance inadaptée : l'héritage de Vichy, Paris, Éditions ouvrières, rééd. 1987, $318 \mathrm{p}$.

CHAUVIÈRE (M.); LENOËL (P.); PIERRE (E.), Protéger l'enfant, Presses universitaires de Rennes, 1996, 183 p. 
DALLHENNE (F.), Le centre d'observation de Paris, Marly-le-Roi, 1950, Arch. CNFE-PJJ, Vaucresson.

FOUCAULT (M.), Surveiller et punir, Paris, Gallimard, 1975, 318 p.

GAILLAC (H.), Les maisons de correction, Paris, Cujas, rééd. 1991, 463 p. MEURILLON, La Maison d'éducation surveillée de Fresnes, 1932, Arch. CNFE-PJJ, Vaucresson.

MAZO (G.), Le centre d'observation et la loi du 27 juillet 1942 relative à l'enfance délinquante, Paris, Van Etten, 1944.

SANCHEZ (C.), Sous les regards de Caïn, Ramonville-Saint-Agne, Érès, 1995, $200 \mathrm{p}$.

SANCHEZ (C.) et TURBELIN (D.), La ferme de Champagne : une histoire au pluriel, Vaucresson, CNFE-PJJ, 1995, $168 \mathrm{p}$.

SANCHEZ (C.), Les éducateurs-observateurs, polycop., 1994, 6 p.

SIGUIER (M.), Droits de regards, Ramonville-Saint-Agne, Érès, 1986, $138 \mathrm{p}$.

SINOIR (G.), Rapport sur les méthodes d'observation à utiliser pour les mineurs délinquants au centre d'accueil, 1945, Arch. CNFE-PJJ, Vaucresson.

Divers témoignages oraux des pionniers de l'Éducation surveillée. 